\title{
El cambio psicoterapéutico en la investigación clínica desde el modelo sistémico
}

\begin{abstract}
RESUMEN: Se destacan los comportamientos etiquetados como sintomáticos que contienen un valor descriptivo y metafórico de las relaciones disfuncionales del contexto significativo del que son efecto oculto e indeseado.

PALABRAS CLAVE: Conocimiento, relación terapéutica, cambio, sistema.
\end{abstract}

SUMMARY: The behaviour labelled as symptoms are considered important from a descriptive and metaphoric point of view and they are a hidden and no wanted results of relations in the significant context.

KEY WORDS: Knowledge, relations therapeutic process, change, system.

La idea de la que partimos y sobre la que venimos trabajando desde hace algunos años (Olabarría, 1991) tiene una doble dirección y a su vez origen en al menos dos autores: por una parte, Pruysser (1976) formula la existencia de condensaciones y/o superposiciones referidas a distintos niveles lógicos que se conjugan en la actividad diagnóstica: definición de entidades clínicas, clasificación, descripción de los procesos psicológicos individuales y el paso inicial del proceso terapéutico. Por otra, Jackson (1960) considera que la rotulación diagnóstica aporta un valor descriptivo y metafórico a las conductas designadas como síntomas, engarzadas éstas en modalidades relacionales características (y tal vez definibles) de cada una de las entidades nosológicas. Precisamente el efecto no deseado del «engarce» de estas determinadas modalidades relacionales serían los síntomas, los trastornos psicopatológicos.

Desde un punto de vista sistémico, hablar de modalidades de relación propias del contexto significativo de un sujeto dado, obliga a su vez a considerar que la percepción y el conocimiento de ese sujeto van a estar modelados por su participación en dicho contexto de relaciones y por las estrategias generales y particulares de los integrantes del mismo. Hacia la modificación de su percepción y, por tanto, de su conocimiento hacia el cambio se dirige la intervención terapéutica.

Señalaré aquí que tomamos la concepción de E. Morin (1986) sobre el conocimiento, concepción en la que distingue entre:

a) El proceso reflexivo de conocimiento que deriva del cogito cartesiano.

b) La capacidad autorreferencial y egocéntrica propia de todo ser vivo.

Esta última se manifiesta operacionalmente en «el cálculo viviente, cálculo del sí mismo, a partir del sí, en función del sí y para el sí». (Morin, 1986, p. 43). Se trata de un conocimiento elemental de carácter estratégico vital, que no se conoce a sí mismo al no disociarse de operaciones concretas de reorganización, autoproducción, reproducción. 
Observemos que esta concepción teórica cuenta con un importante precedente experimental realizado por los biólogos Maturana y Varela (1984) que, estudiando la relatividad de nuestra percepción y conocimiento cuando no sabemos que estamos en él involucrados (ni cómo), proponen un experimento simple en el que llegamos a no ver un punto de una figura en ciertas condiciones, y muestran que en esos momentos «no vemos que no vemos». Podríamos entonces decir que existe un ámbito de desconocimiento del desconocimiento, al menos en ciertas situaciones.

\section{El desconocimiento del desconocimiento y la investigación psicoterapéutica como proceso del tratamiento}

Añadamos a lo anterior que este desconocimiento del desconocimiento forma parte de los modos y procesos de relación de los seres humanos. En el hacer psicoterapéutico encontramos con frecuencia situaciones que dan cuenta de ello. Veamos muy resumidamente una de ellas que nos permitirá ejemplificarlo para pasar de la teoría a la práctica: un caso que se inicia con una demanda promovida por un trastorno de alimentación y que tras la evaluación del sistema familiar, realizamos un contrato terapéutico con una contextualización operativa individual, a sabiendas de que la modulación de su percepción y, por tanto, de su conocimiento, viene condicionada por su contexto familiar, su devenir, por las relaciones y sus reglas, y por las estrategias y las jugadas relacionales en curso.

Se trata de una joven de 26 años, tercera de las cuatro hijas de un matrimonio. Las dos mayores de 35 y 34 años, nacieron durante la emigración de los padres. La mayor nació y las condiciones laborales de los padres llevaron a éstos a valorar las circunstancias desfavorables en las que estaban para realizar adecuadamente las tareas de crianza, por lo que la enviaron con la abuela paterna a España, que se había quedado viuda y sola y era joven, fuerte y capaz.

Cuando nace la segunda, las condiciones de los padres han mejorado, pero la abuela, que se quedaría sola si ahora se llevasen a la mayor, realiza una jugada relacional: propone a su hijo que la traigan para que las dos hermanas no crezcan separadas y así «evitar envidias y celos».

Nuestra paciente ha nacido en España a la vuelta de la emigración y hay una hija más pequeña que ahora tiene 17 años. La paciente manifiesta como contenido de su demanda que se preocupa porque la ropa no le vale, se percibe gorda y poco atractiva; ve a sus amigas «todas con muy buen tipo». Se siente triste. Se queda en casa. Come compulsivamente y luego vomita. Se define como insegura y poco sociable. Cuando «está con gente» no sabe de qué hablar. Sus amigas le 
parecen mucho más interesantes que ella. Es bibliotecaria de formación y de profesión y la primera en casa que ha hecho estudios universitarios, abriendo un camino hasta ese momento inexistente en la familia; la hermana pequeña, «por su edad, todavía no se ha definido». En resumen, presenta un trastorno de la conducta alimentaria de tipo bulímico.

En el modelo sistémico desde el que abordamos la intervención, contamos con distintos instrumentos técnicos en la búsqueda del objetivo psicoterapéutico del cambio, en este caso de la percepción que de sí misma y de lo que la rodea tiene la paciente. Uno de estos instrumentos son las tareas. Así, decidimos pedirle, para el siguiente encuentro, la siguiente: «Debes anotar en las interacciones que establezcas con tus amigos, compañeros, conocidos, etc. lo que creas que personalmente has aportado al buen o mal resultado de ese encuentro».

A la siguiente consulta viene con sus notas. Destacamos que se ha relacionado exitosamente con personas de ambos sexos, de diferentes edades y modalidades de relación, compañeros-as, usuarios de las bibliotecas en la que trabaja, amigas-os, incluso quedó con una prima que ha tenido un problema parecido al suyo y hablaron de ello, pero también de otros temas propios de su edad. Confrontada a estos resultados comenta sorprendida lo que para ella se revela como un comportamiento nuevo y desconocido. «Nunca lo había pensado así y ahora me doy cuenta que esto me pasa fundamentalmente en mi casa, con mi familia, es más el otro día me vi también con mi prima, en presencia de mi hermana mayor y me doy cuenta que estuve todo el tiempo callada y preguntándome qué podía decir, sin encontrar el qué».

Es evidente que sumergida en el conocer propio del cálculo, de carácter estratégico vital, que no se conoce a sí mismo al no disociarse de operaciones concretas..., la realización de la tarea le ha facilitado el acceso a nueva información, le ha producido una confrontación con la hasta entonces establecida y cuyo resultado es que ha generado una nueva percepción de sí: se trata de un resultado cognitivo y emocional y por eso ha podido abrir el proceso reflexivo de conocimiento que deriva del cogito cartesiano y desde y con él, configurar otra percepción abriendo el camino hacia otro conocimiento que consideramos (y buscamos) va a afectar/promover, junto con otras experiencias de similares características, el cambio perceptivo objeto de todo proceso terapéutico y con ello la búsqueda de cambios en las relaciones y en las estrategias relacionales en curso. Cambio que afectará a los receptores a quienes van dirigidas las nuevas estrategias y que no tendrán más remedio que posicionarse, novedosamente, ante ellas.

Cabría, por tanto, distinguir en el ejercicio de los procesos vitales de los seres humanos significaciones y/o representaciones provocadas por líneas de conocimiento propias del cogito y otras provocadas por líneas propias del cálculo 
(¿podríamos pensar que existen ambas líneas en cada producción mental o en muchas de ellas?).

\section{Algunos efectos del cálculo y del cogito}

Esta doble dimensión del conocimiento nos permite acercarnos de una manera «nueva» a otra afirmación de E. Morin (1986): «Los efectos de las acciones son a menudo perversos en relación con sus intenciones». En efecto, desde esta «nueva» perspectiva cabe la posibilidad de que cuando un sujeto actúa en su contexto, no conozca desde el cogito que ha realizado un acto que, además de las razones que el sujeto pueda aportar para su justificación, cuenta con algo determinante y desconocido que es fruto de otro acto dentro de la modalidad estratégica del «cálculo viviente», con sus intenciones y objetivos, tanto en su mantenimiento como en su modificación.

Es el contexto significativo en el que está involucrado el sujeto, es decir, aquél en el que ha generado una estrategia de acción y que está gobernado por un marco de concepciones y reglas propias que sólo ocasionalmente y de manera parcial son reconocidas y tomadas en cuenta (tanto para su cumplimiento como para su transgresión o intentos de modificación).

Este marco aporta las definiciones y valores compartidos por los partícipes de dicho contexto. Definiciones y valores que el sujeto no desconoce, pero los concibe y reproduce como consustanciales a las cosas, situaciones o relaciones. Como si de las cosas mismas emanasen.

Los sujetos que participan de un determinado contexto no se disocian más que ocasional y parcialmente de las operaciones organizativo-relacionales del mismo. En este sentido, se trata de un ámbito primordial del cálculo como modalidad de conocimiento.

La sintomatología evidentemente es un efecto, pero no buscado, ni deseado. De hecho aparece ante el propio sujeto que lo sufre y reproduce, así como ante los partícipes del contexto relacional significativo, como incomprensible y/o ajeno a su posibilidad de dominio, como inevitable. Surge de la oculta metaelección del sujeto en su estrategia de acción. La característica de «elemento natural» que los participantes conceden a las reglas y valores que rigen su contexto, así como el carácter de «intimidad» de las estrategias relacionales que en el mismo se realizan, confieren a los síntomas un carácter de consecuencia ocultamente colectiva, inesperada, desconocida y no buscada: es el efecto del engarce de la estrategia del paciente en su contexto. Una estrategia singular que hemos de investigar. 
Volvamos al ejemplo anteriormente presentado. Nos llama la atención como huella a investigar, por un lado el status de las dos hermanas mayores: solteras, sin novio o proyecto de pareja, sin proyecto de autonomía personal, que viven con los padres. Por otro ella, que, por sus problemas, tiende a quedarse en casa y salir poco. Es fácil identificar dificultades de autonomía y diferenciación, de modo que así se lo planteamos a la paciente. A ella no le llama la atención, «lo ve natural», todos en casa lo ven «natural»... Confrontada a que las hermanas quizás estuvieran desajustadas de lo que, por edad y fase del ciclo vital, les correspondería, (caracterizada por una mayor independencia en la que deberían estar desempeñándose), se queda nuevamente sorprendida. Nunca lo había visto como efecto de un juego relacional con diferentes actores y con diferentes estrategias.

Perfilemos algunos elementos de las estrategias en curso en nuestro ejemplo: veamos en primer lugar la estrategia relacional invasora de su madre. Comenta: «Mi hermana mayor ha tenido novio pero no nos gustaba a ninguno, era un poco particular; a la que menos le gustaba era a mi madre, ya que cada vez que se hablaba del futuro de esta relación que les habría llevado a vivir fuera, mi madre se deprimía diciendo que no lo podría soportar. En cuanto a la segunda, también ha tenido novio, «pero cuando querían salir un tiempo algo más largo... mi madre decía que me tenían que llevar como hermana pequeña».

La psicoterapeuta hace un comentario con tono humorístico: «Ahora tú no tendrás que pasar por el visto bueno de tu madre ni llevar carabina porque gracias a esa pésima imagen que consideras que tienes no sales y todas estamos seguras de que los candidatos no vienen a casa. Bueno... no sólo eliminas este riesgo sino también el de confrontarte a la defensa de tus propios criterios y opiniones... cosa que también sería una novedad porque tus hermanas no lo han hecho. Esa pantalla de la imagen externa sirve para ocultarte a ti y a los demás quién eres tú en realidad». Hipotetizamos que la madre desarrolla una estrategia dirigida al mantenimiento rígido de su condición maternal, sin cambios, evitadora de la emancipación de sus hijas, aquéllas a las que no pudo dar los cuidados de crianza por la decisión de su celoso esposo de llevársela a la emigración y después de entregar las niñas a la abuela paterna como modo de mantener a su esposa a su lado, y al tiempo ofrendar las niñas a su propia madre que quedaba en España sola y viuda garantizando cuidados «adecuados» para sus hijas. Al tiempo para ambos, padre y madre, luchando por la consecución del objetivo de su emigración -mejorar las condiciones económicas familiares-, el ocuparse de la crianza de la hija podía demorar su conquista. Además la madre de esta manera podía obtener «puntos» en la relación con su esposo desde una base de inicio de la relación en que la aceptación por su suegra había venido marcada por la descalificación. La resolución era aparentemente «buena» para todos. Pero justamente el que fuera «buena para todos» hizo 
invisible la pérdida/tristeza por la cesión. La de las hijas cedidas que además unos años después pueden ver el extraordinario interés de su madre por la perfeccionista crianza de las dos menores en la que se vuelca como supermujer. La de la madre que bloqueó el juicio y rotulación de ese ejercicio materno que se otorgó y que no puede expresar ni tal vez para sí misma (incluso bloqueando su percepción), a fin de obtener los «puntos» buscados en la relación con su marido. La del padre que ha de cerrar su percepción sobre los miembros de su familia nuclear, a fin de sostener ampliado el foco de su interés por su familia de origen desde una autonomización deficiente. La salida de las hijas de la casa llevaría a esta madre a tener que afrontar una vida de contacto directo con su esposo hacia el que guarda el resentimiento de no haber definido adecuadamente límites con su familia de origen, generándole el coste de la pérdida de la crianza de sus hijas mayores, provocando así una maternidad deficientemente realizada cuando ella siempre ha buscado exasperadamente su propia perfección. El esposo, con las hijas dentro, tiene contenta y controlada a su mujer y puede hacer su propia vida «hacia fuera». Las hijas mayores «se clavan» ahora en la casa y parecen de este modo querer anular la presencia de la «sustituta» de ellas, la primera de las tres hijas criada por la madre, evitando o dificultando con los poderosos valores filiales que aportan, el proceso de autonomización funcional de la paciente designada. Y la propia paciente designada, que ha establecido una oculta simetría en el ámbito del perfeccionismo con su madre a quien desea vencer, estrategia en la que ocasionalmente cuenta con el resentido apoyo del padre, dentro de las reglas exasperadas que ha ido construyendo la familia.

No dice la terapeuta, al menos todavía, que además su comportamiento y su imagen anoréxicas resultan una buena protesta metafórica contra esta madre que reina en la casa y que ha permitido que su espacio propio como hija en fase de ciclo vital de autonomización, sea cada vez más exiguo ante las hermanas mayores. Al tiempo, el comportamiento y su imagen anoréxicos le permiten también metafóricamente purgar/expiar la culpa por ello.

Es decir, el equipo terapéutico, en la relación terapéutica, va poniendo sobre el tapete las jugadas, las estrategias ocultas, y va favoreciendo con ello el acceso a nuevas percepciones y nuevos conocimientos de la paciente, alimentando el impulso para nuevas exploraciones de experiencia relacional en el contexto significativo, alimentando el cambio.

El elemento de «naturalidad» con el que se ve la dificultad de autonomización, inhibe el comentario espontáneo encerrando los comportamientos en la intimidad. La sintomatología no es un efecto buscado ni deseado. Como vemos, es preciso realizar una investigación terapéutica singular de todo para abordar el cambio, porque se trata del modo como el paciente accede al mismo. 


\section{La investigación y las guías protocolizadas}

Lo anterior no niega, sino que subraya la utilidad de contar con «guías» ante los diferentes cuadros nosológicos que nos marquen el procedimiento de investigación/acción psicoterapéutica y de casos. Nos permitirán conducir con más precisión la imprescindible investigación particular de cada caso. Tal vez ahora se hace preciso decir que una parte importante de la investigación general en psicología clínica y psicoterapia, se centra en este aspecto de unos años a esta parte: los protocolos, aunque desde cada modelo se les otorgue un diferente valor.

Seguramente de este hecho, unido a que la mayor parte de las escuelas psicoterapéuticas conciben su corpus teórico-práctico como una totalidad autorreproductora y excluyente, deviene a nuestro juicio una confusión que es preciso desvelar.

La Investigación (con mayúscula) en psicoterapia se sitúa académicamente en una fase anterior a la ejecución clínica propiamente dicha (siguiendo un modelo de laboratorio químico-farmacéutico). Este modelo marca su función al psicoterapeuta, ajustándola a la tarea de «mirar» para reconocer, intervenir y revalidar el paradigma interventor del que participa. Si se produce una huida en el ejercicio de la psicoterapia como proceso investigador, se aboca a la ceguera, a una rigidificación en la evolución epistémica. El clínico precisa de la condición de investigador de cada caso para generar cambio, así como de la continua ponderación y ajuste epistemológico para sustentar sus hallazgos, corregir sus errores y avanzar en el conocimiento.

La investigación sistémica que se centra a partir del ejercicio clínico en desarrollar hipótesis acerca de las estrategias relacionales características de distintos cuadros nosológicos, estableciendo al mismo tiempo procedimientos de intervención psicoterapéutica para el cambio, podemos concebirla como establecedora de «protocolos/guía» para la investigación posterior particular de cada caso.

Esta investigación particular debe incluir los acontecimientos interactivos, reacciones y estrategias de los partícipes de un contexto, con una perspectiva que incluya el devenir de la historia concreta del mismo para una reconstrucción del proceso interactivo, de las estrategias relacionales de los partícipes, con sus conductas concretas y los elementos emocionales y valorativos, así como sus reglas. Debe considerar los hechos, procesos, crisis y consecuencias esperados en función del ciclo vital y el devenir contextual, también los inesperados y sorpresivos, así como las «resoluciones» $\mathrm{o}$ «salidas» de las crisis y fases y su alcance funcional para el cierre de éstas o su cierre «en falso», que puede jugar un papel posteriormente en la producción sintomatológica.

De ese proceso investigador en el marco de la relación terapéutica, de la progresiva puesta sobre el tapete de las estrategias relacionales, de los efectos de acce- 
so a nuevas percepciones y nuevos conocimientos, a nuevos modos de mirar y ver, a nuevo conocimiento, con el impulso al diseño y realización de nuevas experiencias, estrategias y relaciones, observamos el acceso y devenir del cambio terapéutico.

Los protocolos suponen una guía que señala el procedimiento de «investigación» particular del caso. Pero es esta investigación particular la que va a permitir al paciente el acceso de nuevos elementos al cogito, acceder a otra comprensión epistemológica, uno de los elementos fundamentales en la concepción sistémica del cambio. Esta nueva construcción epistemológica hacia la que se dirige la relación psicoterapéutica precisa de anclajes, de cimientos, de pilares, que han de surgir en la investigación del caso durante el proceso psicoterapéutico. En los «nuevos» elementos, en los «nuevos» valores adjudicados a los hechos, elementos que tienen no sólo valor cognoscitivo, sino también emocional se configura un nuevo entramado epistemológico sobre lo acaecido hasta el presente estableciendo un nuevo sentido, favorecedor del inicio de nuevas estrategias individuales y colectivas en el sistema.

En tanto que investigadores clínicos y en relación con el caso antes citado, debemos desarrollar una hipótesis que dé cuenta de las reacciones y estrategias de acontecimientos «autonomizadores» y de las estrategias de los partícipes en ese contexto, por lo que aquí cabría hipotetizar acerca de la estrategia de la madre en el sistema familiar, el por qué de la misma y el para qué, así también la del padre y su modalidad de autonomización de su familia de origen, los roles jugados por otros elementos del sistema como las hermanas, y la estrategia de la paciente en relación con ello.

Como resultado de esa investigación realizada con el/la paciente designado/a y tal vez con miembros del contexto significativo, de la definición de esas relaciones, de las diferentes estrategias, de sus jugadas en diferentes momentos y fases, habría de configurarse en la paciente un cambio de percepciones y un nuevo entramado epistemológico que habría de dar cuenta tanto del pasado como del aquí y ahora. Orientando hacia un cambio en el percibir y aprehender que ha de permitir generar nuevas estrategias de acción. Se trataría del cambio psicoterapéutico.

\section{Algunas bases epistemológicas de la investigación clínica sistémica}

Para el psicoterapeuta sistémico, la investigación psicoterapéutica y sus objetivos ha de establecerse operativamente en tres niveles interrelacionados: epistémico, de cuadro, de caso. Y ello a través de una relación: investigadora y psicoterapéutica, para el cambio. 
Es evidente que situamos esta reflexión al margen de la polémica sobre el estatuto psicoterapéutico respecto a su condición de ciencia aplicada o práctica artesanal, ubicándonos en la defensa (de la tradición) de una práctica clínica psicoterapéutica que incluye la investigación como elemento consustancial a su existencia para abordar el cambio en tanto éste sería el efecto precisamente de la investigación psicoterapéutica en la relación con el/los pacientes.

Los físicos, a comienzos de este siglo, revisaron las nociones de los métodos en las ciencias de la naturaleza. Los biólogos, al finalizar el siglo, obligan de nuevo a una revisión de las mismas nociones. Estaba ya definido que el concepto de ciencia como descripción objetiva de un mundo sin sujetos, contenía contradicciones disfuncionales. Para superarlas era preciso un «observador» (sujeto). Pero las observaciones no tienen valor absoluto, sino que son relativas al punto de vista del observador y afectan a lo observado hasta anular la esperanza de predicción del observador, al menos en los sistemas complejos autopoiéticos y en su organización específica que los singulariza y donde cobra realidad la autonomía del ser vivo (Maturana y Varela, 1984).

Por tanto, una descripción de un sistema complejo, implica al que lo describe y así se hace preciso una teoría del observador. Pero como sistema vivo que es el observador, debe dar cuenta de sí mismo en la formulación de la teoría. Estamos, pues, en un nuevo estado de cosas en la ciencia. De hecho en la psicoterapia sistémica el terapeuta configura con el paciente y su contexto signicativo/operativo un nuevo sistema marcado por la relación terapéutica que se configura en un espacio/tiempo precisos hacia el cambio. Una relación en la que se juegan diferentes estrategias de los distintos partícipes.

En ese nuevo sistema el subsistema terapéutico observa (en su sentido investigador) e interviene precisamente al contrastar sus hipótesis acerca de lo investigado, lo que busca y tiene el efecto del cambio epistemológico en lo que percibe y ajusta el paciente, permitiéndole así a éste abordar la posibilidad de modificar sus estrategias relacionales que le llevan al síntoma.

El subsistema psicoterapéutico ha de contar con su propia epistemología sobre el movimiento del sistema sobre el que interviene y el protocolo o guía puede favorecer la investigación de cada caso tanto en un sentido económico temporal, como porque permite el establecimiento de distinciones, indicaciones ante el cuadro y ante el caso.

Dicho de otro modo, la vida no puede ser estudiada en laboratorio, sino «en vivo». Se trata de un desafío. Un desafío, que incorpora un cambio de acento en la pregunta científica central, que pasa de ser «por qué» a «cómo».

Una idea fundamental para comprender de qué hablamos al referirnos a la epistemología la aporta Bateson (1972) al señalar que lo que cada cual percibe y cono- 
ce depende de las distinciones que establece: «Las diferentes cualidades abstractas [asignadas] por científicos dados a la corriente de los acontecimientos pueden considerarse como diferentes hábitos de puntuación de la corriente de la experiencia, para obtener algún tipo de coherencia o sentido» (p. 191).

Como nos indica Bradford P. Kenney (1987) esta idea que Watzlawick y Jackson (1981) denominaron «puntuación de la secuencia de los hechos» es similar al concepto de «indicación» de Spencer-Brown, pues un observador (el terapeuta) que traza una distinción, establece concomitantemente una indicación y abre el panorama para la investigación clínica desde otra epistemología, por tanto hacia el cambio.

Se trata de poner en crisis la visión del sujeto acerca del efecto «natural» que emana de las cosas, de la experiencia, de los hechos, para abrirle nuevas posibilidades hacia un nuevo modo de ver desde el que avanzar en el tanteo de nuevas experiencias, hacia una nueva epistemología, una nueva manera de ver, ensayos de nuevas estrategias, de todo lo cual el efecto será el cambio. El/la paciente, o el otro subsistema del sistema terapéutico, ha de confirmar con sus ensayos progresivos de experiencia que los efectos no son «naturales», son productos, son creencias, construidos en un marco de relaciones estratégicas susceptibles de modificación.

El estudio de los procedimientos por los que la gente puntúa su experiencia, que considera «natural», es un método para identificar su epistemología. Sus pautas de puntuación presuponen ciertas premisas para establecer distinciones. Dicho de otro modo, el reordenamiento de los marcos de referencia («reencuadre», Watzlawick, 1981) crea nuevas realidades en un sujeto dado.

Consecuentemente con todo esto, la relación psicoterapéutica sistémica pretende organizar un contexto, y uno de cuyos objetivos fundamentales es modificar las premisas (que actúan en los sujetos como «naturales», no pensadas o construidas) de la puntuación, que organizan y estructuran la realidad, las relaciones en el contexto dado, con el fin de alcanzar el cambio. En este sentido cabe hablar de una epistemología clínica.

El terapeuta comprende la experiencia de un individuo observando de qué manera puntúa su contexto, la organización de las relaciones y hechos del mismo. En función de esta puntuación, el individuo está definiendo su estrategia relacional.

Dado que, como Selvini y Prata (1990) mostraron -siguiendo a Bateson-, el individuo y/o la familia acuden a consulta de psicoterapia con su propia epistemología, el terapeuta ha de contar con una epistemología sobre su epistemología. 
Contar entonces con una guía (protocolo) para la investigación clínica de cada caso en función de la modalidad relacional específica característica de los contextos significativos de los pacientes con una determinada patología, resulta de interés. Se trata de una modalidad de epistemología clínica. Un tipo de comprensión referida a las líneas de relaciones que establecen, los modos en que los pacientes y los partícipes de su contexto adquieren y sostienen sus peculiares formas de conocer el mundo y de relacionarse estratégicamente en él. Y ese modo de investigación, con sus sucesivas «puestas sobre el tapete» en la relación terapéutica, es la base del cambio terapéutico que, desde un punto de vista sistémico, no puede dejar de estar entrelazado.

\section{A modo de conclusión}

La reconstrucción por el actor del sentido de su acción relacional en su contexto, la apropiación de su resultado a través del nuevo significado y la modificación de estrategias relacionales, generadoras del cambio, justifica así la investigación clínica propia de la acción psicoterapéutica sistémica.

¿Qué aporta a la epistemología sistémica el instrumento clínico que es un protocolo?, ¿qué aporta al mejor comprender del sujeto que hace una petición de ayuda?, ¿qué aporta para facilitar el cambio terapéutico? Podríamos decir que es, sin duda, un instrumento propio del cogito, que pertenece al ámbito de la psicología como ciencia que generaliza (psicología explicativa, siguiendo a Dilthey) permitiendo una construcción ordenada de los modelos relacionales característicos de cuadros nosológicos específicos, definiendo pautas de actuación unificadas en o para aquellas demandas que comparten las características que definen a cada uno de esos cuadros. Al tiempo, son instrumentos de investigación en tanto permiten enfrentar, conocer, aprehender, la singularidad del entramado de relaciones y conductas que, engarzadas, dieron como efecto no deseado los síntomas (situándose en el ámbito de la psicología descriptiva de Dilthey). Los protocolos facilitan neutralizar la mirada, a veces proyectiva, del observador/psicoterapeuta que, en tanto ser vivo, participa de lo observado.

El uso de estas modalidades de «protocolos» desde la perspectiva sistémica no supone la aplicación rígida y «de receta», sino una ayuda para pensar «en y con el paciente de forma más libre» (Tizón, 1992). Nos permiten acercarnos al sí, a partir del sí y para el sí de un sujeto partícipe activo de un contexto.

La investigación clínica en el modelo sistémico resulta así no sólo necesaria, sino complementaria de la investigación básica, como elemento central del proceso psicoterapéutico para la reconstrucción epistemológica del sujeto concreto en su contexto y para su acceso al cambio. 


\section{BIBLIOGRAFÍA}

(1) ANDERSEn, T., El equipo reflexivo. Diálogos y diálogos sobre los diálogos, Barcelona, Gedisa, 1994.

(2) ANDERSON, H.; Goolishian, H., «Human Systems as Linguistic Systems: Evolving Ideas about the Implications for Theory and Practice», Family Process, 1988, 27, pp. 371-393.

(3) Anderson, H.; Goolishian, H., «El experto es el cliente: La ignorancia como enfoque terapéutico», en McName, S.; Gergen, K. J., La terapia como construcción social, Barcelona, Paidós, 1992.

(4) Bateson, G., Pasos hacia una ecología de la mente, Buenos Aires, Carlos Lohlé, 1976.

(5) Bateson, G., Una unidad sagrada. Pasos ulteriores hacia una ecología de la mente, Barcelona, Gedisa, 1993.

(6) Berger, P.; Luckman, T., La construcción social de la realidad, Buenos Aires, Amorrortu, 1966.

(7) Bowen, M., De la familia al individuo. La diferenciación del sí mismo en el sistema familiar, Barcelona, Paidós, 1991.

(8) DiltheY, W., Introducción a las ciencias del espíritu, Madrid, Alianza, 1980.

(9) HALEY, J., «Descripción interaccional de la esquizofrenia», en JACKSON, D. (comp.), Comunicación, familia y matrimonio, Buenos Aires, Nueva Visión, pp. 183-206, 1977.

(10) Harre, R.; Clarke, D.; De Carlo, N., Motivos y mecanismos. Introducción a la psicología de la acción, Barcelona, Paidós, 1989.

(11) JACKSON, D., Etiología de la esquizofrenia, Buenos Aires, Amorrortu, 1960.

(12) Kenney, B. P., Estética del cambio, Buenos Aires, Paidós, 1987.

(13) Maturana, H.; Varela, F., Las bases biológicas del entendimiento humano. El árbol del conocimiento, Santiago de Chile, Universitaria, 1984.

(14) Maturana, H.; Varela, F., El árbol del conocimiento, Santiago de Chile, Universitaria, 1986.

(15) Maturana, H. R., La realidad: ¿Objetiva o construida? II. Fundamentos biológicos del conocimiento, México, Anthropos, 1996.

(16) Maturana, H. R., «El observador en su observación. Tres conferencias y una reflexión sobre la biología del conocer, del amor y la constitución sistémica de la identidad del ser», Systémica, 1997, 2, pp. 13-52.

(17) Morin, E., El método: La naturaleza de la naturaleza, Madrid, Cátedra, 1986.

(18) OlabarRía, B., «Reflexiones para una epistemología de la relación entre la locura y el quehacer técnico. Hacia una hipótesis relacional de la esquizofrenia desencadenada en adultos autónomos», Clínica y Salud, 1991, 2, 3.

(19) Pinsof, W.; Wynne, L., «Toward Progress Research: Closing the Gap Between Family Therapy», Journal of Family Therapy, 2000, 1, pp. 45-62.

(20) Prata, G., El niño que seguía la barca, Bilbao, Desclèe de Brouwer, 2001.

(21) Pruysser, P. W.; Menninger, K., «Language Pitfalls in Diagnostic Thought and Work», en Pruysser Diagnosis and the Difference it Makes, Nueva York, Menninger Foundation, 1976.

(22) SElvini, M., y otros, Los juegos psicóticos en la familia, Barcelona, Paidós, 1990.

(23) Selvini, M., y otros, Paradoja y contraparadoja. Un nuevo modelo en la terapia de familia a transacción esquizofrénica, Buenos Aires, ACE, 1982.

(24) Simmel, G., El individuo y la libertad, Barcelona, Península, 1986.

(25) SLUZKI, C., «Transformations: A Blueprint for Narrative Changes in Therapy», Family Process, 1992, 31, pp. 217-230. 
(26) Tizón García, J. L., Atención primaria y salud mental en Atención Primaria, Barcelona, Doyma, 1992.

(27) Viaro, M.; LeONARDI, P., «Le insubordinazioni», Terapia Familiare, 1982, 12, pp. 41-63.

(28) Watzlawick, P., ¿Es real la realidad?, Buenos Aires, Paidós, 1976.

(29) Watzlawick, P.; Beavin, J.; Jackson, D., Teoría de la comunicación humana, Barcelona, Herder, 1981.

(30) Watzlawick, P., El lenguaje del cambio, Barcelona, Herder, 1989.

(31) Watzlawick, P., La realidad inventada, Barcelona, Gedisa, 1994.

* Begoña Olabarría, psicóloga clínica y psicoterapeuta sistémica. Dpto. Atención al Ciudadano. Ayuntamiento de Madrid; Pilar Vázquez, psicóloga clínica y psicoterapeuta sistémica. Centro Salud Mental de Móstoles. Comunidad de Madrid.

Correspondencia: olabarriagmb@munimadrid.es; p_vazquezserrano@yahoo.es

** Fecha de recepción: 04-VII-2006. 